$17^{\text {th }}$ International Congress of Metrology, 02006 (2015)

DOI: $10.1051 /$ metrology $/ 201502006$

(C) Owned by the authors, published by EDP Sciences, 2015

\title{
COMPARAISONS INTERLABORATOIRES EN ETALONNAGE ET EN ESSAI - RETOUR D’EXPERIENCE
}

\author{
Soraya Amarouche \\ Laboratoire National de Métrologie et d'Essais (LNE) \\ 1, rue Gaston Boissier 75724 Paris cedex 15, France. \\ soraya.amarouche@lne.fr
}

\begin{abstract}
Demonstration of performance in testing and calibration becomes a pre-requisite within the framework of a peers recognition (MRA: Mutual Recognition Arrangement) either within the framework of an accreditation ISO 17025 [ 1 ] or ISO 15189 [ 2 ] or other standards. Participation in interlaboratory comparisons is the most relevant and independent means to estimate capacity in performing measurements. The challenge for participants is to opt for programs of interlaboratory comparisons which address their objectives as well as competent organizers of comparisons. The organization of interlaboratory comparisons is recognized as being a full competence with reference to the ISO 17043 standard and adapted methodological tools. In France, recognition and visibility of organizers has been on-going for years and is continuously improving.
\end{abstract}

\section{Les comparaisons interlaboratoires}

\subsection{Qu'est-ce qu'une comparaison interlaboratoires}

Le terme de comparaison interlaboratoires est très général et couvre plusieurs pratiques qu'il faut distinguer. La définition de la comparaison interlaboratoires est l'organisation, l'exécution et l'exploitation de mesurages, d'essais ou d'étalonnages sur des objets semblables (échantillons, étalons, matériaux ou solutions de référence, ...), par au moins deux laboratoires (participants) différents dans des conditions prédéterminées. En fonction de l'objectif, la mise en œuvre d'une comparaison interlaboratoires dans son organisation, exécution et exploitation est différente. Il existe actuellement 3 objectifs distincts :

- Evaluer les performances (ou l'aptitude) des participants (référentiel : NF ISO 17043[3]) Il s'agit d'évaluer et de démontrer l'aptitude des participants à réaliser la mesure (essais ou étalonnages). Chaque participant met alors en œuvre sa méthode de mesure habituelle, normalisée ou non, sur les supports proposés.

- Estimer l'exactitude (justesse et fidélité) d'une méthode de mesure (NF ISO 5725 [4]). Il s'agit d'établir la performance d'une méthode de mesure. Les participants mettent alors en œuvre exactement une même méthode de mesure.

- Attribuer une valeur consensuelle à une caractéristique d'un matériau ou échantillon ou solution (Guide ISO 34 [5]). Il s'agit alors d'assigner une valeur de référence à un matériau. Les laboratoires participants doivent être experts dans la détermination de la caractéristique concernée.

Ces 3 objectifs possibles sont résumés dans la figure 1 ci-dessous :

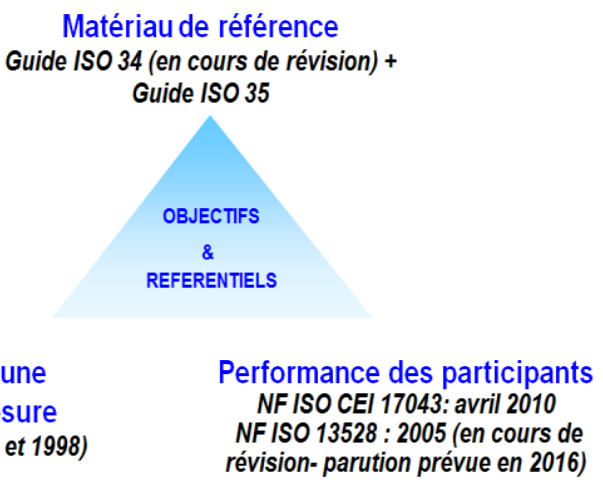

Figure 1: Objectifs \& Référentiels d'une comparaison interlaboratoires

L'organisation de comparaisons interlaboratoires est une activité qui nécessite de multiples compétences. Il n'est

soraya.amarouche@lne.fr 
plus acceptable de réaliser une comparaison sans un minimum de préparation et de formation sous peine de ne pouvoir exploiter et obtenir les informations recherchées. Beaucoup d'apprentis organisateurs ont en fait l'amère expérience et avec eux les participants qui n'ont jamais reçu leur rapport, faute d'exploitation possible.

Nous aborderons dans cet article les comparaisons interlaboratoires organisées dans le but d'évaluer la performance des laboratoires (ou essais d'aptitude) et qui constituent la majorité des comparaisons interlaboratoires recensées. Nous ferons le point sur les compétences nécessaires et les circuits disponibles.

\subsection{Essais d'aptitude - Performance des participants}

Une comparaison interlaboratoires (CIL) 'type essai d'aptitude' est une ensemble d'étapes clés, de la conception à l'envoi du rapport, qui peuvent se schématiser par le processus décrit dans la figure 2 cidessous.

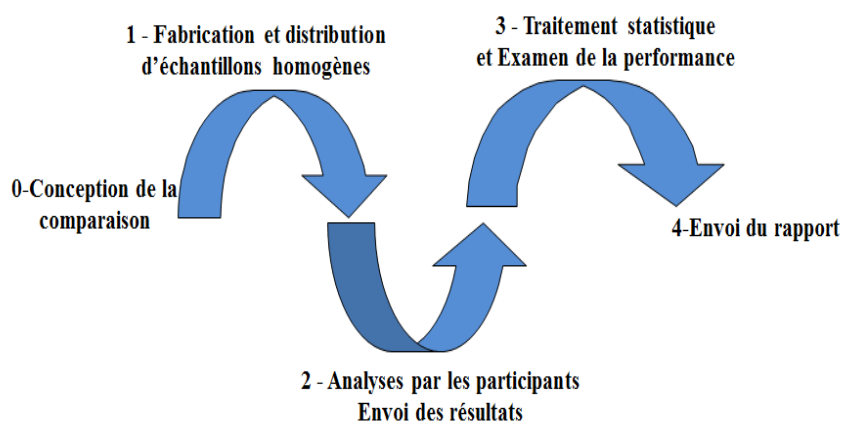

Figure 2 : Processus d'une comparaison interlaboratoires

Ce processus demande à l'organisateur d'avoir dans son équipe, un certain nombre de compétence qui sont les suivantes :

- Qualité

- Techniques (connaître le domaine dans lequel est organisé la CIL)

- Choix de l'objet de la CIL

- Evaluation de l'objet (homogénéité, stabilité)

- $\quad$ Transport (maîtrise)

- Mesure (démonstration de l'homogénéité, stabilité)

- Exploitation statistique et choix des indicateurs

- Interprétation...

Les participants aux comparaisons interlaboratoires sont responsables du choix de la campagne et de l'organisateur considérés un fournisseur. Le participant doit donc s'assurer à partir des informations fournies par l'organisateur de l'adéquation de la comparaison à son besoin ainsi que de sa compétence. Ces informations doivent figurer dans les documents d'appel à participation à la comparaison, ainsi que dans les documents remis par l'organisateur.

Une analyse de certains points est à réaliser pour être dans les objectifs visés:

\section{-La caractéristique :}

Le participant doit s'assurer que la méthode de mesure qu'il met en œuvre est en cohérence avec la caractéristique exploitée par l'organisateur.

Exemple : matière grasse, correction d'étalonnage en température, métaux, HAP, Radionucléides émetteurs $\gamma, \ldots$

\section{-Le support de la comparaison :}

Il se présente sous différentes formes échantillon liquide solide (lait cru, sang, eau d'alimentation, plaque de bois, tissu, grains de blé, farine,..), étalon voyageur (capteurs, cales, masses,...) objet (vélo, four, ...). Il sera envoyé simultanément ou en circulation à tous les participants. L'organisateur doit en vérifier l'homogénéité, la stabilité (dérive) sur la durée de la comparaison. Il existe des méthodes statistiques qui évaluent l'homogénéité et la stabilité selon des critères prédéfinis (NF ISO 13528 [6])

La caractéristique et le support vont permettre de définir si le participant est en mesure de participer à la comparaison. L'organisateur n'indique pas toujours les méthodes éligibles à participer, ce n'est pas son domaine de compétence.

\section{-L'intervalle de mesure ou les points de mesure :}

En étalonnage, les points d'étalonnage à réaliser sont indiqués: exemple de $-\left[-30^{\circ} \mathrm{C} ; 350^{\circ} \mathrm{C}\right]$ avec les points suivants : $-30^{\circ} \mathrm{C} ; 0^{\circ} \mathrm{C} ; 50^{\circ} \mathrm{C} ; 150 \mathrm{C} ; 250^{\circ} \mathrm{C}$; $350^{\circ} \mathrm{C}$ ). Dans les essais, il n'est pas en général indiqué de valeur afin d'évaluer le participant dans son aptitude à détecter ou pas la présence de l'élément et à le quantifier, comme dans la routine. L'organisateur peut mettre des leurres dans les échantillons afin d'éviter la collusion. Les niveaux de mesures sont cohérents avec les méthodes en routine des participants.

\section{-La valeur assignée et l'écart-type d'aptitude :}

L'organisateur doit indiquer avant la comparaison comment il obtiendra la valeur assignée. Ce choix n'est pas anodin car elle intervient dans l'évaluation de l'aptitude du participant. L'expertise de l'organisateur réside dans sa compétence à avoir une valeur assignée représentative qui peut être :

- Une valeur de référence $X_{\text {Réf }}$ (étalonnage à haut niveau, matériau de référence certifié, méthode de référence,...)- elle est dans ce cas obtenue avant le début de la comparaison et indépendante des résultats des participants. $\mathrm{Ce}$ cas n'est pas toujours possible dans le cas des essais.

- Une valeur consensuelle $X_{\text {Ass }}$ déterminée à partir des résultats d'une partie des participants ou de tous les participants. Il existe différents modes 
de calcul que l'organisateur appliquera en évaluant la pertinence en fonction de la comparaison. Des méthodes de calcul non exhaustives sont proposées dans la norme NF ISO 13528 [6]. Les organisateurs appliquent généralement une méthode de calcul qui permet par itération d'obtenir une moyenne robuste et un écart-type robuste qui ne sont pas impactés par les valeurs aberrantes (Algorithme A - NF ISO 13528 [6]).

\section{-La statistique de performance :}

L'organisateur doit indiquer le mode d'évaluation de la performance du participant, objectif principal de ce type de comparaison. Il existe différentes statistiques de performance qui sont listées dans le tableau cidessous

\begin{tabular}{|c|c|c|}
\hline \multicolumn{3}{|c|}{ STATISTIQUES DE PERFORMANCE } \\
\hline Différence ou biais & \multicolumn{2}{|l|}{$D=X_{l a b}-X_{a s s}$} \\
\hline Différence en \% & \multicolumn{2}{|c|}{$D_{\%}=100 \times\left(X_{l a b}-X_{a s s}\right)$} \\
\hline Ecart normalisé $\mathrm{E}_{\mathrm{N}}$ & \multicolumn{2}{|c|}{$E_{N}=\frac{\left(X_{l a b}-X_{r e f f}\right)}{\sqrt{U_{l a b}^{2}+U_{r e f}^{2}}}$} \\
\hline \multicolumn{2}{|l|}{ Score Z } & Zscore $=\frac{\left(X_{\text {lab }}-X_{\text {ass }}\right)}{\sigma_{\text {apt }}}$ \\
\hline \multicolumn{3}{|c|}{ Score Z' $\quad Z^{\prime}=\frac{\left(X_{\text {lab }}-X_{a s s}\right)}{\sqrt{\sigma_{a p t}{ }^{2}+u_{a s s}^{2}}}$} \\
\hline Score Zeta & & $\frac{\left(X_{l a b}-X_{a s s}\right)}{\sqrt{u_{l a b}^{2}+u_{a s s}^{2}}}$ \\
\hline
\end{tabular}

Tableau 1 : Les statistiques de performance

Avec,

Xréf: valeur de référence

Xlab : valeur du participant

Ulab: incertitude élargie associée à la valeur du participant

Uréf : incertitude élargie associée à la valeur de la référence

$\sigma_{\text {apt }}$ : écart-type d'aptitude

L'écart-type d'aptitude $\sigma_{a p t}$ est soit fixée par l'organisateur, soit calculé à partir des résultats de tous les participants. La valeur de ce paramètre va dans le cas, par exemple du score $Z$, jouer un rôle plus ou moins contraignant pour le participant. L'objectif étant pour l'organisateur de l'évaluer ou le fixer pour qu'il soit représentatif de la dispersion admissible toutes méthodes de mesure confondues.

Il est classique dans le cas des essais ou des analyses de présenter la performance du participant par un score $\mathbf{Z}$ dont les conclusions sont définies par les critères suivants : $|z| \leq 2 \quad$ L'aptitude du participant est satisfaisante

$2<|z|<3 \quad$ L'aptitude du participant est discutable

$|z| \geq 3 \quad$ L'aptitude du participant est insatisfaisante et le participant doit en rechercher la cause.

Le mode d'évaluation de la performance le plus pertinent dans le cas d'une comparaison interlaboratoires en métrologie est l'écart normalisé $\left(E_{N}\right)$ qui est l'écart entre la valeur du participant et la valeur de référence et au dénominateur la racine de la somme des incertitudes aux carrées de chacune des valeurs. Si la valeur de référence est mal définie et que son incertitude est grande au regard des participants, elle ne permet pas d'être utilisée pour évaluer l'aptitude. Il faut donc être vigilant aux propositions de valeur de référence et leur incertitude associée fournies par l'organisateur. Les conclusions pour l'écart normalisé sont définies par les critères suivants :

$\left|E_{n}\right| \leq 1 \quad$ Aptitude satisfaisante

$\left|E_{n}\right|>1 \quad$ Aptitude non satisfaisante

Remarque : Si l'objectif du participant est mal défini, choix d'un circuit inadapté peut conduire à ne pas détecter des problèmes. Avec par exemple un objet soumis à essai de résolution insuffisante (non discriminant).

\section{-Rapport (livrable)}

Le rapport est un document autoporteur qui doit contenir toutes informations nécessaires sur le processus de mise en œuvre de la comparaison interlaboratoires. Avec :

- L'organisateur ou les organisateurs

- La description du ou des supports de la comparaison

- Les résultats des participants

- Des informations sur la stabilité, homogénéité du support

- Le calcul de la valeur assignée et son incertitude de mesure

- Le calcul de l'écart-type d'aptitude

- L'évaluation de la performance

- Des conseils,... 


\section{Comment trouver un organisateur de comparaison Interlaboratoires?}

Les comparaisons interlaboratoires sont organisées par différentes structures qui peuvent être :

- des associations qui souhaitent répondre aux besoins d'évaluer les performances de ces adhérents

- Le Laboratoire national de métrologie dans sa mission de référence nationale en métrologie (LNE en France) et autres laboratoires national de référence (LNR)

- des entreprises privées...

En métrologie, le LNE (laboratoires national de métrologie et d'essais) organise régulièrement dans le cadre de ses missions des comparaisons interlaboratoires en métrologie.

http://www.lne.fr/fr/metrologie/comparaisonsinterlaboratoires-campagnes.asp.

\begin{tabular}{|l|l|l|l|l|}
\hline Exemple :programme $2012-2013$ & \multicolumn{3}{|l|}{} \\
\hline Domaine & Support & $\begin{array}{l}\text { Date de } \\
\text { lancement }\end{array}$ & Statut \\
\hline Métrologie des Masses & $\begin{array}{l}\text { Etalon de masse de 1 } \\
\text { Tonne }\end{array}$ & Mai 2013 & Terminée \\
\hline Métrologie Temps/ Fréquence & $\begin{array}{l}\text { Etalon de fréquence àa } \\
\text { rubidium }\end{array}$ & $\begin{array}{l}\text { Début } \\
2013\end{array}$ & Terminée \\
\hline Métrologie desmasses / pesage & Balances (IPFNA) & $\begin{array}{l}\text { Janvier } \\
2013\end{array}$ & Terminée \\
\hline Métrologie dimensionnelle & Calesétalons & $\begin{array}{l}\text { Septembre } \\
2012\end{array}$ & Terminée \\
\hline Métrologie électrique & Shunt & Fin 2012 & Terminée \\
\hline
\end{tabular}

La position du LNE dans la chaîne de traçabilité lui permet de fournir une valeur de référence et son incertitude associée indépendante et de meilleur niveau que tous les participants. De plus, il possède une grande expérience dans l'organisation et l'exploitation de comparaisons nationales et internationales (key comparisons).

Exemple : CIL national en étalonnage d'un couple thermoélectrique de type $\mathrm{S}$ au point $800^{\circ} \mathrm{C}$ :

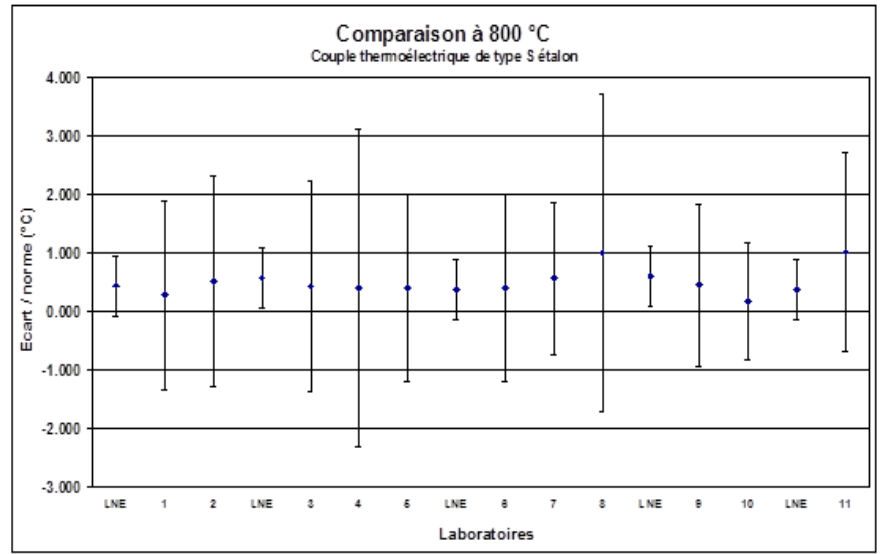

Le LNE assure la valeur de référence et un suivi de l'étalon de transfert avec un retour régulier au LNE au cours de la comparaison (voir figure ci-desus)

L'ouverture à l'accréditation par le Cofrac (comité français d'accréditation) pour les organisateurs de comparaisons interlaboratoires (essais d'aptitude) date de 2004 (www.cofrac.fr). Le nombre d'organisateurs d'essais d'aptitude accrédités est actuellement de 20, principalement dans les essais, avec une nette progression ces 4 dernières années. (Figure 3 ).

\section{Matériau de référence (MR) \\ Accréditation Cofrac \\ Producteur de matériaux de référence}

\section{Reconnaissance des compétences}

\section{Performance d'une méthode}

Pas d'accréditation

Figure 3 : Accréditations Cofrac

$\mathrm{Au}$ niveau international, un groupe de travail d'EA (European cooperation for Accreditation) travaille à la mise en place d'un accord de reconnaissance de l'accréditation des OCIL (organisateur de comparaisons interlaboratoires parmi les pays européens membres d'EA.

Pour avoir l'ensemble des organisateurs de comparaisons interlaboratoires, il existe une base de données avec inscription volontaire qui est hébergée par le BAMfederal Institute for Materials Research and Testing (www.eptis.bam.de) 
Exemple de résultats d'une

CIL :

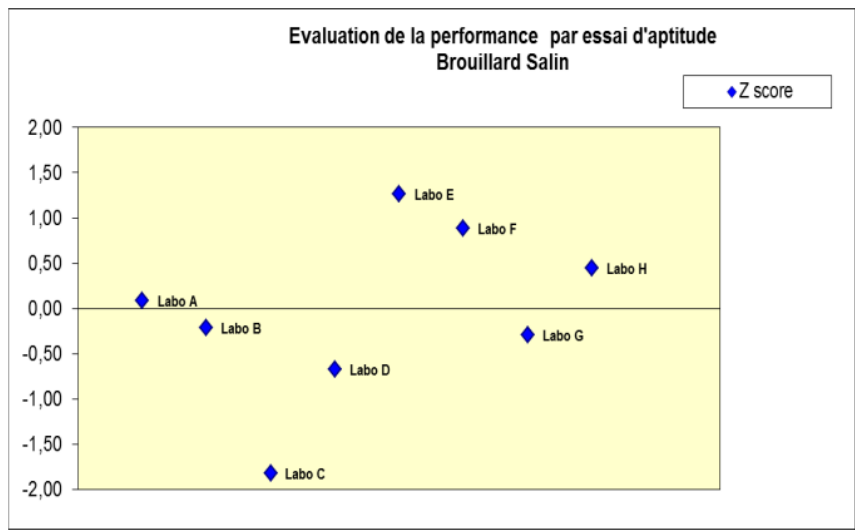

\section{Conclusion}

Il y a une prise de conscience depuis ces dernières années sur l'apport positif de participer à des comparaisons interlaboratoires avec un accès à des informations qu'il faut prendre le temps d'exploiter (court terme et long terme). La confiance dans l'organisation et l'exploitation des comparaisons interlaboratoires repose sur un ensemble de critères que le participant doit évaluer avant de participer afin que son choix réponde à ses objectifs. Le développement de l'offre doit prendre en compte la montée en exigence des participants.

\section{Références}

[1] NF EN ISO/CEI 17025 (sept.2005) Exigences générales concernant la compétence des laboratoires d'étalonnages et d'essais.

[2] NF EN ISO 15189 (Décembre 2012)

Exigences concernant la qualité et la compétence Laboratoires d'analyses de biologie médicale

[3] NF EN ISO/CEI 17043 (Avril 2010)

Évaluation de la conformité - Exigences générales concernant les essais d'aptitude

[4] NF ISO 5725-2 (1994)

Exactitude (justesse et fidélité) des résultats et méthodes de mesure. Partie 2 Méthode de base pour la détermination de la répétabilité et de la reproductibilité d'une méthode de mesure normalisée

[5] FD ISO GUIDE 34 Mai 2010

Exigences générales pour la compétence des producteurs de matériaux de référence

[6] NF ISO 13528 Décembre 2005

Méthodes statistiques utilisées dans les essais d'aptitude par comparaisons interlaboratoires 